\title{
Performance Evaluation of the V-BLAST System under Correlated Fading Channels
}

\author{
Trung Q. Duong(*), Hoang Trang (**), Een K. Hong (*), Sung Y. Lee(*) \\ (*)School of Electronics and Information. Kyung Hee University - Republic of Korea \\ dqtrung@oslab.khu.ac.kr \\ (**)HoChiMinh City University of Technology - Vietnam
}

\begin{abstract}
The MIMO system (multiple antennas at the transmitter and receiver) is capable of very high theoretical capacities. As an important space-time code, V-BLAST (Vertical-Bell Lab Layered SpaceTime) code has been researched recently. However, most research in this area has assumed that the antennas are decorrelated to each other at both ends. Unfortunately, in practice, this assumption is not satisfied. This paper introduces a model for correlated channel matrix, and as a result the degradation of BER performance can be simulated without the constraint of the number of antennas. In addition, the stochastic property of SNR of the received signal is demonstrated, and the analytical upper bound of the average probability of error for Zero-Forcing V-BLAST under correlated Rayleigh fading channels is derived. The simulation will verify the analysis.
\end{abstract}

\section{Introduction}

It has been shown in recent research that the deployment of multiple antennas at both sides of a transmitter and receiver provides a larger capacity compared to single antenna systems [1], [2], and [3]. As an important space-time code, V-BLAST (VerticalBell Lab Layered Space-Time) code was proposed in [3]. This high capacity can be obtained under the assumption that the channels at the transmitter and receiver are uncorrelated. However, in a real fading environment, signals are not independent The effect of correlated fading has been investigated in [4], [8], [9], and [10]. The performance loss due to correlation in V-BLAST was investigated in [5]. An experimental study verified that MIMO radio channels suffer from correlated fading in [11]. Existing works attain no general channel matrix model under correlated fading for the V-BLAST system. This paper introduces a correlated channel matrix model, and therefore it is easily applied in simulation. Furthermore, we derive the stochastic property of SNR of the received signal and the upper bound of error probability is given.

This paper is organized as follows. In section II, the V-BLAST system and Zero-Forcing criterion are briefly reviewed. In section III, the general model for the correlated fading channel matrix is introduced. In section IV, we investigate the stochastic property of the SNR of received signal. The results are compared by simulation in section $\mathrm{V}$ and a concluding remark is given in section $\mathrm{VI}$

\section{Zero-Forcing V-BLAST System Overview}

In this section, the V-BLAST architecture and Zero-Forcing are reviewed. The V-BLAST system is considered with $n_{T}$ transmitted and $n_{R} \geq n_{T}$ received antennas. The data is demultiplexed in $n_{T}$ data sub-streams of equal length (called layers). These sub-streams are mapped into M-PSK or M-QAM symbols $t_{1}, t_{2}, \ldots, t_{n_{T}}$ and simultaneously transmitted over $n_{T}$ antennas. In order to outline the V-BLAST system, one time slot of the time-discrete complex base band model is examined. Let $t=\left[t_{1} \ldots t_{n_{T}}\right]^{T}$ define the $n_{T} \times 1$ vector of transmit symbols, then the

- This research was supported by the MIC(Ministry of Information and Communication), Korea, under the ITRC(Information Technology Research Center) support program supervised by the IITA(Institute of Information Technology Assessment) 
corresponding $n_{R} \times 1$ vector of receive symbols $r=\left[r_{1} \ldots r_{n_{R}}\right]^{T}$ is given by

$r=H \cdot t+n$

In $^{1}$ (1), $n=\left[n_{1} \ldots n_{n_{R}}\right]^{T}$ stands for the white Gaussian noise of variance $\sigma_{n}^{2}$ observed at the $n_{R}$ receive antennas while the average transmit power of each antenna is normalized to one i.e.

$E\left\{t t^{H}\right\}=I_{n_{T}}$, and $E\left\{n n^{H}\right\}=\sigma_{n}^{2} I_{n_{R}}$

The $n_{R} \times n_{T}$ channel matrix $\mathrm{H}$

$H=\left(\begin{array}{ccc}h_{1,1} & \cdots & h_{1, n_{T}} \\ \vdots & \ddots & \vdots \\ h_{n_{R}, 1} & \cdots & h_{n_{R}, n_{T}}\end{array}\right)$

includes complex fading gains $h_{j i}$ expressing the tap gains between transmit antenna $i$ and receive antenna j

In [3] the decorrelating matrix $\mathrm{D}$ in the ZeroForcing criterion was given by

$D_{\text {ZF }}=H^{+}=\left(H^{H} H\right)^{-1} H^{H}$

At the $i-t h$ detection step, the receive vector is linearly weighted with the nulling vector which is the $i-t h$ row of $D$, and the result is given by:

$y_{i}=(D)_{i} \cdot r=(D)_{i}(H \cdot t+n)$

$y_{i}=t_{i}+\widetilde{n_{i}}$

$y_{i}$ is considered as the decision statistic for the $i-t h$ sub-stream $\left(t_{i}\right)$, where $\widetilde{n_{i}}=D_{i} \cdot n$ is the noise enhancement. By using the quantization operation $Q[$.$] appropriately, the i-t h$ sub-stream can be likely estimated

$\hat{t_{i}}=Q\left[y_{i}\right]$

\section{MIMO Correlated Fading Channel Matrix Model}

\footnotetext{
${ }^{1}$ In this paper, (.) ${ }^{\mathrm{T}}$ and $(.)^{\mathrm{H}}$ represents for the matrix transposition
} and Hermitian transposition, in that order. $\mathrm{I}_{\mathrm{a}}$ denotes the $\mathrm{a} \times \mathrm{a}$ identity matrix
In this section, we introduce a general model for a channel matrix under the correlated fading environment. This model can be easily used in simulation to validate analysis.

In [9], the spatial fading correlation matrix for a Rayleigh fading channel $H$ is denoted as

$R=E\left[\operatorname{vec}(H) \operatorname{vec}(H)^{H}\right]$

If $H$ is a $n_{R} \times n_{T}$ matrix, then $\operatorname{vec}(H)$ defines $n_{R} n_{T} \times 1$ vector by stacking the columns of $H$ under each other.

In richly-scattered propagation environments we can assume that the correlation among received antennas is independent of the correlation between transmitted antennas (and vice versa) i.e. only immediate surroundings of the antenna array impose the correlation between array elements and have no impact on the correlations observed between the elements of the array at the other end of the link. Therefore, $R$ can be described in [11] as follows

$R=R_{T X} \otimes R_{R X}$

with $\otimes$ defining the Kronecker product; $R_{T X}$ and $R_{R X}$ represent the transmitted and received correlation matrix, respectively. It is very clear that $R$, $R_{T X}$ and $R_{R X}$ are symmetrical complex correlation matrices i.e. Hermitian matrices. According to [12], we can write

$R=P X P^{H}$

where $P$ is a unitary matrix, and $X$ is a diagonal matrix. Applying the square-root transformation to $X$, so from (10) it follows that

$R=P X^{1 / 2} X^{1 / 2} P^{H}$

Since the transpose of a diagonal matrix is also itself, we get

$R=P X^{1 / 2} X^{H / 2} P^{H}$

$R=\left(P X^{1 / 2}\right)\left(P X^{1 / 2}\right)^{H}=R^{1 / 2} R^{H / 2}$

The equation (13) also holds for $R_{T X}$ and $R_{R X}$. Substituting (13) for (9), we attain

$R=\left(R_{T X}^{1 / 2} R_{T X}^{H / 2}\right) \otimes\left(R_{R X}^{1 / 2} R_{R X}^{H / 2}\right)$

By using some properties of the Kronecker product in [13], we can write (14) as follows 
$R=\left(R_{T X}^{1 / 2} \otimes R_{R X}^{1 / 2}\right)\left(R_{T X}^{H / 2} \otimes R_{R X}^{H / 2}\right)$

since $(A \otimes B)(C \otimes D)=(A C \otimes B D)$

$R=\left(R_{T X}^{1 / 2} \otimes R_{R X}^{1 / 2}\right)\left(R_{T X}^{1 / 2} \otimes R_{R X}^{1 / 2}\right)^{H}$

since $\left(A^{H} \otimes B^{H}\right)=(A \otimes B)^{H}$

Let $\tilde{H}$ be $n_{R} \times n_{T}$ independent and identically distributed (iid) Rayleigh matrix with complex Gaussian elements with zero means and unit variances i.e. $E\left\{\operatorname{vec}(\tilde{H}) \operatorname{vec}(\tilde{H})^{H}\right\}=I \quad$. Then the correlation matrix in (15) can be described as follow

$$
R=E\left\{\left(R_{T X}^{1 / 2} \otimes R_{R X}^{1 / 2}\right) \operatorname{vec}(\tilde{H}) \operatorname{vec}(\tilde{H})^{H}\left(R_{T X}^{1 / 2} \otimes R_{R X}^{1 / 2}\right)^{H}\right\}
$$

$R=E\left\{\left(R_{I X}^{1 / 2} \otimes R_{R X}^{1 / 2}\right) \operatorname{vec}(\tilde{H})\left[\left(R_{T X}^{1 / 2} \otimes R_{R X}^{1 / 2}\right) \operatorname{vec}(\tilde{H})\right]^{H}\right\}$

since vec $(A B C)=\left(C^{H} \otimes A\right) \operatorname{vec}(B)$, we get

$$
R=E\left\{\operatorname{vec}\left(R_{R X}^{1 / 2} \tilde{H} R_{T X}^{H / 2}\right)\left[\operatorname{vec}\left(R_{R X}^{1 / 2} \tilde{H} R_{T X}^{H / 2}\right)\right]^{H}\right\}
$$

From (8) and (18), the normalized channel matrix can be written as

$$
H=R_{R X}^{1 / 2} \tilde{H} R_{T X}^{H / 2}
$$

This analytic result is equal to the correlation model introduced in [10]. The spatial correlation of a narrow-band flat fading channel depends on the physical parameters of the model, which include antenna distance, antenna arrangement, angle spread, and angle of arrival [9]. However, it is very complicated to investigate all these parameters. Furthermore, it is hard to cover a wide range of bestcase to worst-case scenarios [14]. In [8], an easy-touse exponential correlation matrix model was introduced. In addition, Zelst and et al have exposed a similar model in [14]. These models are completely compatible with the measured model in [11].

For exponential model, the components of a correlation matrix are given by

$\rho_{i j}=\rho^{|i-j|}$

where $\rho$ is the correlation coefficient of neighboring branches. In the case of uniform illumination, the correlation coefficient between two adjacent antennas is [15]

$\rho=J_{0}\left(2 \pi \frac{d}{\lambda_{c}}\right)$

where $J_{0}$ is the zeroth order Bessel function, $\lambda_{c}$ is the carrier wavelength, and $d$ is antenna space. The general form of transmitted and received correlation matrices are given, respectively.

$$
R_{T X}=\left[\begin{array}{ccccc}
1 & \rho_{T X} & \rho_{T X}^{2} & \cdots & \rho_{T X}^{n_{T}-1} \\
\rho_{T X} & 1 & \rho_{T X} & \ddots & \vdots \\
\rho_{T X}^{2} & \rho_{T X} & 1 & \ddots & \rho_{T X}^{2} \\
\vdots & \ddots & \ddots & \ddots & \rho_{T X} \\
\rho_{T X}^{n_{T}-1} & \cdots & \rho_{T X}^{2} & \rho_{T X} & 1
\end{array}\right]
$$

$$
R_{R X}=\left[\begin{array}{ccccc}
1 & \rho_{R X} & \rho_{R X}^{2} & \cdots & \rho_{R X}^{n_{R}-1} \\
\rho_{R X} & 1 & \rho_{R X} & \ddots & \vdots \\
\rho_{R X}^{2} & \rho_{R X} & 1 & \ddots & \rho_{R X}^{2} \\
\vdots & \ddots & \ddots & \ddots & \rho_{R X} \\
\rho_{R X}^{n_{T}-1} & \cdots & \rho_{R X}^{2} & \rho_{R X} & 1
\end{array}\right]
$$

where $\rho_{T X}$ and $\rho_{R X}$ stand for the transmitted and received correlation coefficient, in that order. These correlated matrices will be applied in the next section to demonstrate the upper bound of error probability and in the simulation to verify the analysis.

\section{Upper bound of average error probability.}

In this section, we study the statistical property of the SNR of the received signal, thereby verifying the average error probability. When applying the ZeroForcing criterion, the signal to noise ratio of $k-t h$ received substream is given by

$$
S N R_{k}=\frac{S N R_{0}}{\left[A^{-1}\right]_{k k}}
$$

where $A=H^{H} H,\left[A^{-1}\right]_{k k}$ is the $k k-t h$ element of matrix $A^{-1}$, and $S N R_{0}=\frac{E_{S}}{N_{0}}$

According to the inverse matrix property we have: 


$$
\left[A^{-1}\right]_{k k}=(-1)^{k+k} \frac{\operatorname{det}\left(\left[A_{k k}\right]\right)}{\operatorname{det}(A)}
$$

where $\left[A_{k k}\right]$ is the minor of matrix $\mathrm{A}$ by deleting the $k$-th row and $k-t h$ column of the matrix A.

Hence,

$$
S N R_{k}=S N R_{0} \frac{\operatorname{det}(A)}{\operatorname{det}\left(\left[A_{k k}\right]\right)}
$$

It is very important to determine the statistical property of the random variable $\gamma_{k}=\frac{\operatorname{det}(A)}{\operatorname{det}\left(\left[A_{k k}\right]\right)}$

It can be described as a positive quadratic form

$\gamma_{k}=H_{k}^{H} \tilde{H} H_{k}$

where $\tilde{H}$ is an Hermitian and non negative matrix. Using unitary transformation in $\tilde{H}$, we may express

$\gamma_{k}=H_{k}^{H} \tilde{H} H_{k}=H_{k}^{H} U^{H} \Lambda U H_{k}$

where $\mathrm{U}$ is a unitary matrix and $\Lambda$ is $\operatorname{diag}\left(\lambda_{1} \lambda_{2} \ldots \lambda_{n_{R}}\right)$, with $\lambda_{i}$ being an eigenvalue of $\tilde{H}$. Let $Z=U H_{k}$, and hence $z^{H}=H_{k}^{H} U^{H}$, so $\gamma_{k}$ is described as

$\gamma_{k}=z^{H} \Lambda z$

or in the matrix form

$\gamma_{k}=\left[\begin{array}{llll}z_{1}^{*} & z_{2}^{*} & \ldots & z_{n_{R}}^{*}\end{array}\right]\left[\begin{array}{cccc}\lambda_{1} & \ldots & \ldots & 0 \\ 0 & \lambda & \ldots & 0 \\ \ldots & \ldots & \ldots & \ldots \\ 0 & \ldots & \ldots & \lambda_{n_{R}}\end{array}\right]\left[\begin{array}{c}z_{1} \\ . \\ . \\ z_{n_{R}}\end{array}\right](30)$

$\gamma_{k}=\sum_{i=1}^{n_{R}} \lambda_{i}\left|z_{i}\right|^{2}$

Since $H_{k}$ is a complex Gaussian vector, and $\mathrm{U}$ is a unitary matrix, $H_{k}$ and $z$ possess identical statistics. $\gamma_{k}$ is a sum-squares of Gaussian random variables, thus it is a chi-square random variable. In order to identify the degree of freedom of $\gamma_{k}$, we have to determine the eigenvalues of $\tilde{H}$. In [7], Winters and et al have discovered and proved that $\tilde{H}$ has
$n_{T}-1$ eigenvalues equal to zero and $n_{R}-n_{T}+1$ eigenvalues equal to 1 .

Using this evaluation, we can express $\gamma_{k}$ as below

$\gamma_{k}=\sum_{j=1}^{n_{R}-n_{T}+1} \lambda_{j}\left|z_{j}\right|^{2}$

Then, the SNR of the $k-t h$ received subtream is a chi-square error with the degree of freedom $2\left(n_{R}-n_{T}+1\right)$ and variance $\sigma_{k}^{2}$. Then it is not difficult to prove that the mean of SNR on the $k-t h$ received substream is

$E\left(S N R_{k}\right)=2\left(n_{R}-n_{T}+1\right) S N R_{0} \sigma_{k}^{2}$

This result is the same as that in [5] and [6] by using the Schur complement and Wishar distribution. If using random detection order, the received signal is individually detected i.e. the average symbol probability can be obtained by averaging the instantaneous probability of error over all the SNRs as in [5]

$\bar{P}_{e, k} \leq E\left(S N R_{k}\right)\left[N_{e} \exp \left(-\frac{d_{\min }^{2} S N R_{k}}{4}\right)\right]$

where $N_{e}$ is the average number of nearest neighbors, and $d_{\text {min }}$ is the minimum distance between any two symbol constellation.

In this paper we only consider the correlation among the transmitted antennas; hence we attain $H=\tilde{H} R_{T X}^{H / 2}$

In addition, the variance of the $k-t h$ substream is given in [5]

$\sigma_{k}^{2}=\frac{1}{2\left[R_{T X}^{-1}\right]_{k k}}$

Substituting (36) and (33) for (34), the average error probability can be obtained as follows

$\bar{P}_{e, k} \leq \frac{N_{e}}{2}\left(\frac{1}{\frac{2 \sigma_{k}^{2} d_{\min }^{2}}{4}+1}\right)^{n_{R}-n_{T}+1}$

The result indicates that there will be no diversity gain if the number of transmitted antennas is equal to the number of received antennas. 


\section{Simulation Results}

In the simulation, we investigate the bit error rates (BER) of V-BLAST system for the Zero-Forcing criterion deploying uncoded BPSK modulation through flat correlated Rayleigh fading channel. Fig. 1 shows the correlation effect at the transmitter on BER performance for a Zero-Forcing V-BLAST system $\left(n_{T}=4, n_{R}=5\right)$. BER performance of uncorrelated case is reduced $3.5 \mathrm{~dB}$ for correlation coefficient equal to 0.7 and nearly the same for correlation coefficient equal to 0.2 . The impact of correlation at the receiver is given in Fig. 2. It shows the same results as in Fig. 1. Furthermore, when the number of transmitted antennas is equivalent to the number of received antennas we can get no diversity gain.

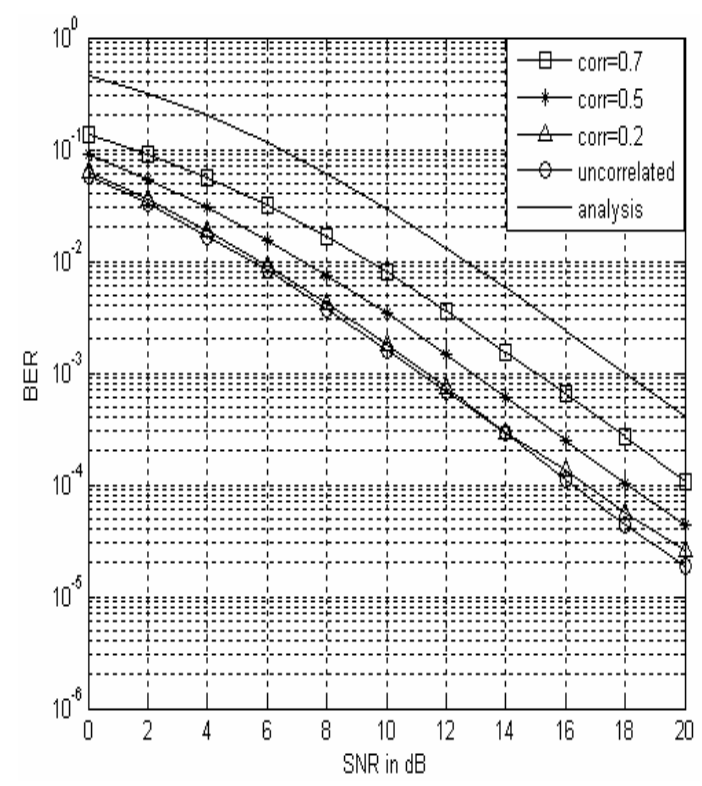

Fig. 1. Zero-Forcing V-BLAST $\left(n_{T}=4, n_{R}=5\right)$, correlation among the transmitted antennas

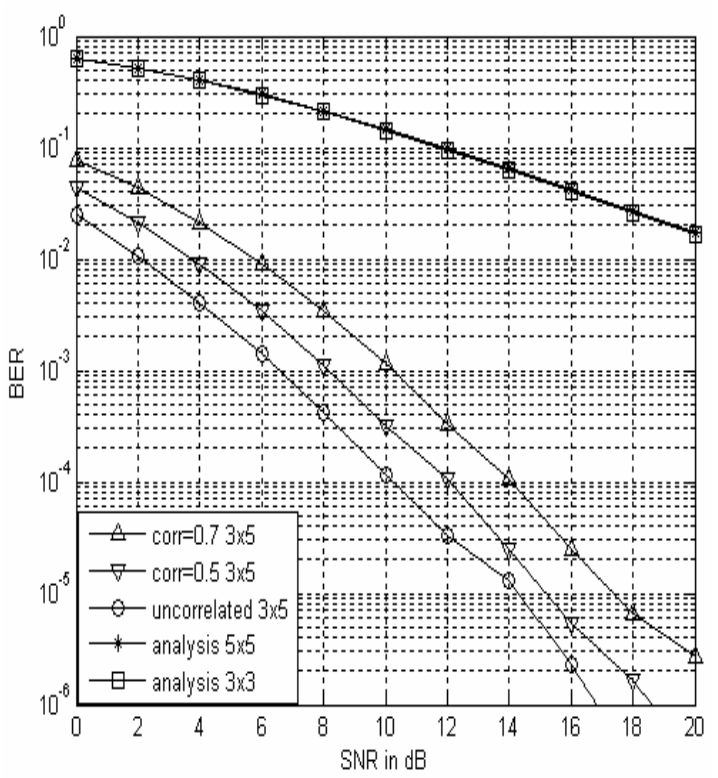

Fig. 2. Zero-Forcing V-BLAST $\left(n_{T}=3, n_{R}=5\right)$, correlation among the received antennas

\section{Conclusions and Discussion}

We have introduced a general model of channel matrix for a V-BLAST system under a correlated Rayleigh fading environment. This model is easily applied in simulation to verify the average error probability analysis due to the stochastic property of the SNR of the received signal.

\section{Acknowledgment}

The authors would like to express special thanks to Dr. A. Van Zelst for many helpful discussions, and address profound thanks to Professor Kevin D. Lee for his guidance and support.

\section{References}

[1] G.J.Foschini. "Layered Space-Time Architecture for Wireless Communication in a Fading Environment When Using Multiple Antennas,” Bell Laboratories Technical Journal, 1(2): 41-59, autumn 1996.

[2] G.J.Foschini. and M.J.Gans. "On the Limits of Wireless Communication in a Fading Environment When Using Multiple Antennas," Wireless Personal Communications, vol. 6, pp. 311-355, 1998.

[3] P. W. Wolniansky. , G.J.Foschini, G. D. Golden, and R. A. Valenzuela. "V-BLAST: An architecture for realizing very high data-rates over the rich-scattering wireless channel,”, Proc. ISSSE 98, Pisa, Italia, September 1998. 
[4] Gesbert, D.; Bolcskei, H.; Gore, D.A.; Paulraj, .J, "Outdoor MIMO wireless channels: models and performance prediction.” IEEE Trans. Commun., vol. 50, no. 12, Dec. 2002, pp. 1926-1934.

[5] Xin Li; Zaiping Nie, "Performance losses in V-BLAST due to correlation." IEEE Antennas and Wireless Propagation Letters, vol. 3, 2004, pp. 291-294.

[6] Gore, D.; Heath, R.W., Jr.; Paulraj, A, “On performance of the Zero-Forcing receiver in presence of transmit correlation”, Information Theory Proceedings, IEEE International Symposium, 2002, pp 159.

[7] Winters, J.H.; Salz, J.; Gitlin, R.D, “The impact of antenna diversity on the capacity of wireless communication systems.” IEEE Trans. Commun., vol. 42, Feb/Mar/Apr 1994, pp. 1740-1751.

[8] Loyka S. L, "Channel capacity of MIMO architecture using the exponential correlation matrix", IEEE Communications Letters, vol. 5, no. 9, September 2001, pp. 369-371.

[9] Da-Shan Shiu, G. J. Foschini, M. J. Gans and J. M. Kahn, "Fading correlation and its effect on the capacity of multielement antenna systems", IEEE Transactions on Communications, vol. 48, no. 3, March 2000, pp. 502-513.

[10] Chizhik, D.; Rashid-Farrokhi, F.; Ling, J.; Lozano, A. "Effect of antenna separation on the capacity of BLAST in correlated channels”, IEEE Communications Letters, vol. 4, no. 11 , November 200, pp. 337-339

[11] Kermoal, J.P.; Schumacher, L.; Pedersen, K.I.; Mogensen, P.E.; Frederiksen, F, “A stochastic MIMO radio channel model with experimental validation” IEEE Journal on Communications, vol. 20, no. 6, Aug. 2002, pp. 12111226

[12] Brinkmann Klotz, Linear algebra and analytic geometry, Addison-Wesley 1971.

[13] Alexander Graham, Kronecker products and matrix calculus with applications, Halsted Press, John Wiley and Sons, NY, 1981.

[14] A. van Zelst and J.S. Hammerschmidt, "A Single Coefficient Spatial Correlation Model for Multiple-Intput Multiple-Output (MIMO) Radio Channels", 27th General Assembly of the International Union of Radio Science (URSI), Maastricht, the Netherlands, August 2002.

[15] W. C. Jakes, Microwave Mobile Communications, New

$\begin{array}{llll}\text { York, NY: } & \text { Wiley, } & 1974\end{array}$ 